Additionally, Charlson's comorbidity index was measured and was about 1,38 in observed patients' group (12/1).

Methods and results.

We used the retrospective data of the 303 patients operated in 2015-2016 years in Central Hospital of Oil workers, Bak1, Azerbaijan. Clinical characteristics (age, gender, cigarette, alcohol consumption, body mass index, systolic and diastolic blood pressure, heart rate, degree of arterial hypertension, functional classes and duration of ischemic heart disease, comorbidities, the results of laboratory and instrumental examinations) has been obtained from the histories of the patients.The classification of the Global Health Organisation was used for division of the patients for the groups according to the levels of the systolic and dyastolic arterial pressure. The level of the stable exertional was assessed in accordance with Canada Cardiovascular Society classification. Patients were distributed on gradation of clinical characteristics.

The results showed exertional angina- $44,9 \%$, unstable angina- $55,1 \%$,arterial hypertension - 43,2\%,diabetes mellitus - 42,9\%,fatty liver - 27,7\%,pulmonary diseases $-19,8 \%$,kidney diseases $-11,2 \%$,prostate diseases $-9,6 \%$,cholecystitis $-10,2 \%$,peripheral artery disease$3,3 \%$,gastric or duodenal ulcer in $1 \%$ of preoperative patients. Charlson's comorbidity index was 3,5 in medium:less than 3 points-24,1+-2,5\%, 3-5,0 points 33,7+-2,7\%,5,1 and more points - in $42,2+-2,8 \%$ of the observed patient group.

\title{
Conclusions
}

Results of the research showed that preoperative CABG patients in our research had a lot of different comorbidities and high comorbidity index.

\section{Moskalets O.V. \\ Effects of extracorporeal blood purification techniques on cytokine levels in patients with gram-negative sepsis}

\author{
Moscow Regional Research Clinical Institute \\ named after M.F.Vladimirskij
}

(Russia, Moscow)

doi: 10.18411/scienceconf-05-2019-27

idsp: scienceconf-05-2019-27

Sepsis is one of the main causes of death in critically ill patients. According to the literature, mortality ranges from 40 to $70 \%$, and in sepsis caused by gram-negative microflora it is twice as high[1,2]. The key element in the pathogenesis of gram-negative sepsis is the release of a large amount of bacterial endotoxin - lipopolysaccharide (LPS). The response to the penetration of endotoxin into the bloodstream is the production of proinflammatory cytokines (TNF- $\alpha$, IL-1, IL-6). However, if the defense mechanisms fail, it becomes uncontrolled, as a result a systemic inflammatory reaction occurs, accompanied by impaired coagulation, increased endotoxicosis, endothelial dysfunction, tissue damage and the development of multiple organ failure $[1,6,6]$. Thus, the efficient removal of endotoxin from patient's blood is very important in the clinical medicine and in the last years interest around the use of extracorporeal blood purification techniques has increased. The choice of a specific method of purification and the moment for its implementation still remains the subject of scientific discussions $[8,9,11]$. One of the promising methods that have emerged in recent years is the coupled plasma filtration adsorption (CPFA). It was established that the sorbent is capable of binding a whole range of cytokines, in particular IL-1, IL-8, IL-6, gamma IFN, TNF- $\alpha$, as well as complement components C3a / C5a and endotoxin of gram-negative bacteria [7, 10]. Another promising method is the selective adsorption of LPS using a specially synthesized polypeptide with a strong selective potential for LPS. There are first reports that the use of this method in the early stages of the development of endotoxemia contribute to blocking the trigger signal and, accordingly, prevent cytokine hyperproduction and the development of multiple organ failure $[2,4]$.

The aim of this preliminary study was to evaluate the levels of proinflammatory cytokines (TNF- $\alpha$, IL-1, IL-6), soluble receptors for cytokines (an antagonist of the IL-1 receptor (pa IL-1), soluble IL-6 receptor (pRIL- 6), soluble receptor of TNF- $\alpha(\operatorname{rrFNO}-\alpha)$ ) and neopterin 
in sera of patients with gram-negative sepsis before and after CPFA procedures and selective lipopolysaccharide adsorbtion using ALTECO LPS Adsorber columns.

Patients and methods. 14 patients ( 8 men, 6 women) with gram-negative sepsis between the ages of 32 and 64 years were included in the study. In all cases the source of infection was intra-abdominal focus. The average score on the scale of organ failure associated with sepsis (SOFA) was $7.1 \pm 0.8$ points. All patients before admission to the department were carried out surgical interventions of varying degrees of complexity. Comprehensive intensive care was carried out in accordance with the standards of treatment of sepsis. Microbiological examination of the intended source of infection in all patients revealed microbial associations, in which gram-negative microflora prevailed. The patients were randomly divided into 2 groups of 7 people, in one group CPFA was performed, in the other - selective lipopolysaccharide adsorbtion using ALTECO LPS Adsorber columns. The determination of the initial levesl of the studied markers was carried out on the 15-21st day from the moment of admission to the intensive care unit. Blood was taken before and immediately after each procedure. The number of procedures ranged from 1 to 3 , depending on the severity of the patient's condition.

Results. Significant individual variations of baseline serum cytokine levels were found. In most cases (12 patients), the concentrations of IL-1, TNF- $\alpha$ corresponded to normal values or slightly exceeded, probably due to the fact that the studies were conducted in the late periods of sepsis. It was noted a significant (2-4 p.) increase in the concentrations of serum IL-6, as well as pp IL-1,6, TNF- $\alpha$, which is also typical for the late phase of systemic inflammation. Neopterin levels significantly (5-10 times) exceeded normal values. Statistically significant differences in the concentrations of cytokines, soluble receptors of cytokines and neopterin before and after blood purification procedures were not obtained. There was a tendency to a decrease in the level of neopterin and an increase in the concentration of RRIL-6.

Conclusion Extracorporeal detoxification methods have an ambiguous effect on the production of pro-inflammatory cytokines, which may be due to the individual features of immunoreactivity and the phase of the systemic inflammatory response. To identify statistically significant trends, research is needed on larger samples and more homogeneous groups of patients.

\section{****}

1. Emirova K.,Tolstova E.M., Kagan D.M., Orlova M.Yu., Abaseeva T.Yu., $\quad$ Pankratenko $\quad$ T.E., Shpikalova I.Yu. Hemolytic uremic syndrome associated with shiga-toxin-producing Esherichia coli // Nephrology, 2016, Vol.20, no.2, pp.18-32 (in Russ.).

2. Karpman D., LooseL., Tati R., Arvidsson I. Haemolitic uraemic syndrome // J. of Int. Medicine, 2017, Vol.281, no.2, pp.123-148.

3. Page A.V., Liles C.W. Biomarkers of endothelial activation/dysfunction in infectious disease // Virulence, 2013, Vol.4, no.6, pp.507-516.

4. Ito N., Hataya H., Saida K. et al. Efficacy and safety of eculisumab in childhood atypical haemolytic uremic syndrome in Japan. // Clin.Exp.Nephrol., 2016, Vol.20, no.2, pp.265-272.

5. Rodrigues E., Barrios C., Soller G. Should eculizumab be continued in patients with atypical hemolytic uremic syndrome? //Clin. Kydney J., 2017,no.3, pp.3230-3332.

\section{Sultanova M.M. \\ The course and resolution of post-traumatic endophthalmitis in children \\ Azerbaijan State Advanced Training Institute for Doctors named after A. Aliyev, Department of ophthalmology}

doi: 10.18411/scienceconf-05-2019-28

idsp: scienceconf-05-2019-28

\section{Key words: eye injury, endophthalmitis, children.}

In this paper, we would like to share our algorithm of action in post-traumatic endophthalmitis in children, as well as the results obtained by us in the clinic.

Objective: to analyze the treatment of eye injury complicated by endophthalmitis in children. 